\title{
Conceptual Learning of Sciences Supported by Java Compliant technologies
}

\section{S. Divjak}

Faculty of Computer and Information Science, University of Ljubljana

Trzaska 25, 1000 Ljubljana, Slovenia

e-mail: sasa@fri.uni-lj.si

\begin{abstract}
The paper presents the concept of reusability of experiments prepared with CoLoS authoring tools and converted into a Java environment. This permits increased platform independence and a possible usage in a distributed environment. The original CoLoS experiments are running on Motif compliant platforms which are normally used on workstations with the UNIX operating system. These experiments are converted into applets which can be used as normal components of hypertext based lessons. In such a way original CoLoS authoring tools are still useful for the development of new experiments that can be used on platforms which are most popular in the schools. The concept is further extended with the possibilities offered by the local networks and the Internet. The additional tools for distance learning and distributed groupwork are developed.
\end{abstract}

Keywords

Simulation, Physics, Distance learning, Java 


\section{INTRODUCTION}

CoLoS (COnceptual Learning Of Science) is a consortium founded in 1988, initially composed of research teams from western European universities. Today the research teams from the following universities represent CoLoS (in alphabetical order): Balearic Islands, Berlin, Cambridge, Cottbus, Delft, Edinburgh, Genova, Oxford, Kiel, Las Palmas, Ljubljana, Lyon, Madrid, Maribor, Murcia, Paris, StPetersburg. Fields of interest of various CoLoS research groups are: physics, chemistry, electrical engineering, mechanical engineering, computer science.

The major goal of this consortium is to encourage and co-ordinate the development of teaching methods to improve the knowledge and understanding of fundamental concepts with a special focus on intuitive and qualitative approaches, more particularly in the scientific and technical fields.

The general CoLoS objectives are: to enhance the teaching of science by using innovative and highly interactive software; to explore the new ways of using computer applications in scientific learning; to develop an intuitive understanding and feel for science in students.

One of the methods that has been developed by Col os is based on the mimicry of nature and its fundamental principles instead of solving mathematical equations. The computer is used for the visualisation of molecular or atomic behaviour. The teacher or students can interact with the simulated system and in this way obtain a better understanding. From the technical point of view this is achieved through 2D or 3D visualisation of simulated world equipped with the needed control buttons, sliders and other possible interacting components.

During the first years of the CoLoS activity more than 60 applications have been developed, the common platforms being powerful workstations with the UNIX operating system and with good computational and graphical characteristics. The development of new experiments has been enabled by means of authoring tools like xdev and xyZET. Xdev is in fact a program generator that enables the creation of new applications through a user- friendly graphical interface. XyZET is both an authoring tool for the development of new experiments and a simulator for their execution. Both tools have been upgraded with the possibility for the remote control of experiments through the commands that can be included and activated in hypertext- based lessons.

The expansion of the Internet and the appearance of Java have permitted the development of platform independent applications. This technological trend has influenced the strategy of CoLoS. The need for reusability of already developed courseware demanded the development of the additional software tools which are described in this paper. 


\section{REUSABILITY OF EXPERIMENTS PREPARED BY XYZET}

XyZET is an interactive, graphically oriented simulation tool that permits the presentation of objects and structures in 3D. The basic building elements of these objects are particles that are defined by their mass, charge, initial position and velocities. These particles can be connected with springs. In such a way more complex, non-rigid bodies can be presented. Different internal and external forces influence the particles in the system. Their behaviour can be observed during the animation. The basic phenomena from the domain of mechanics and electricity can be explored (kinematics, conservation of energy and momentum, Hook's law, gravity, charges, field lines and equipotential planes).

The conceptual learning of the particular phenomena can be achieved by incremental building of simple and then increasingly more complex bodies and structures and by experimentation with various physical parameters. The teaching scenario can be included in accompanying and interacting hypertext. In such a way complete courses in the domains of mechanics and electricity have been created. In the course of mechanics the student first achieves the understanding of the coordinate system, of constant and accelerated motion. He continues with the experiments explaining the basic mechanical laws and ends with an advanced study of various pendulums. These examples are simple but didactically well selected and explained.

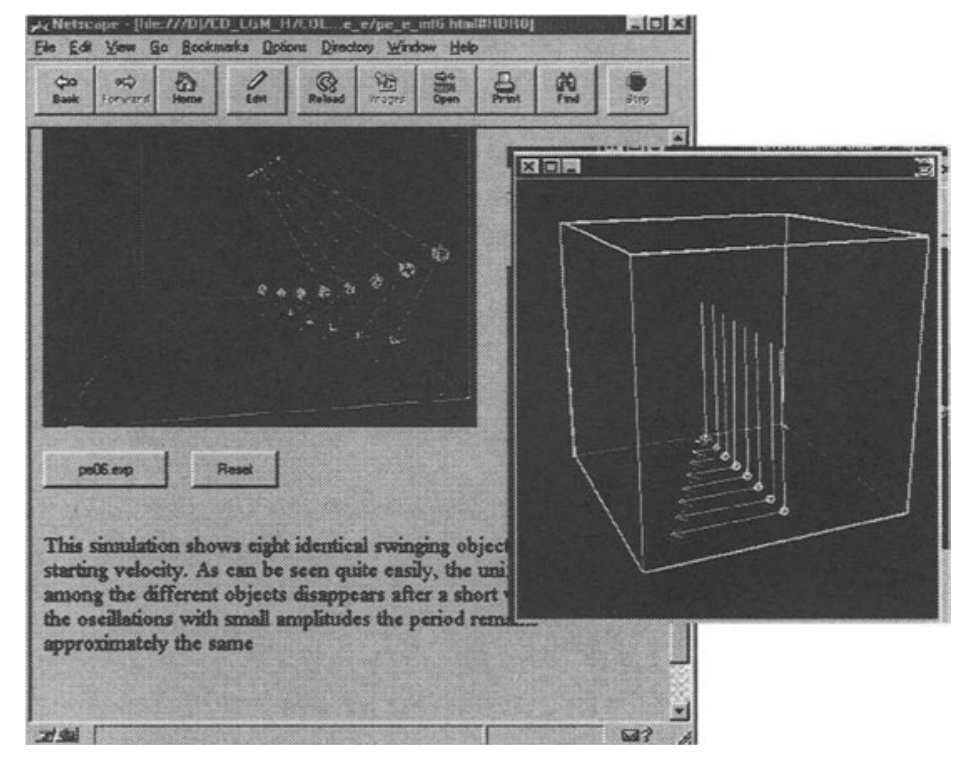

Figure 1. Screenshot with a hypertext lesson and an interacting experiment 
The gallery of more than 200 experiments includes some extremelly complex examples with bodies consisting of more than 100 interconnected particles.

In order to permit a platform- independent visualisation of these experiments a viewer has been developed in the form of a Java applet. This applet is represented as a button which is integrated in the hypertext- based lessons. Activating the button, a visualized 3D world in a separate frame appears. The parameters of the experiment can be controlled by means of buttons, sliders and other controls that are located in a user-friendly way in the hypertext. Figure 1 Presents a screenshot with a simple physical experiment explaining the dependence between period and amplitude.

The simulation can be suspended, reset or restarted by activating the corresponding buttons. According to the selected mouse mode (rotate, zoom, pan) the function of the mouse changes. Dragging the mouse over the frame the viewpoint to the visualized world is changed. The user can activate numerical and graphical monitors which correspond to some observed variables. Graphical monitors permit the visualisation or better time-dependent plot of velocities, forces, accelerations and other variables of selected objects. The numerical monitors permit the modification of some significant properties of the selected objects.

\section{CONVERTING EXAMPLES PREPARED WITH XDEV}

Xdev is another frequently used authoring $\operatorname{CoLoS}$ tool. It permits the design of new experiments by means of a user friendly interface. The building components of developed experiments are widgets. The most signifficant widgets are $2 \mathrm{D}$ and $3 \mathrm{D}$ worlds which can contain the basic (graphical) primitives (normally particles, points and lines). These two types of widgets are used for the visualisation of the models of the physical experiments. Observed experiments can be controlled by the usual buttons, sliders and similar controls which are represented as widgets.

$\mathrm{Xdev}$ is in fact a program generator with a graphically oriented user interface. The basic xdev frame is shown on the figure 2 .

A creative teacher can design new experiments by inserting and modifying the basic components in a defined frame. He can describe the behaviour of the particles and other primitives in the included 2D and 3D worlds by specifying the algorithms of the simulated experiment in a simplified C-code. The result of $x d e v$ is a Motif-compliant program. Experiments prepared with xdev can be combined with hypertext and remotely controlled as is done by xyZET. 


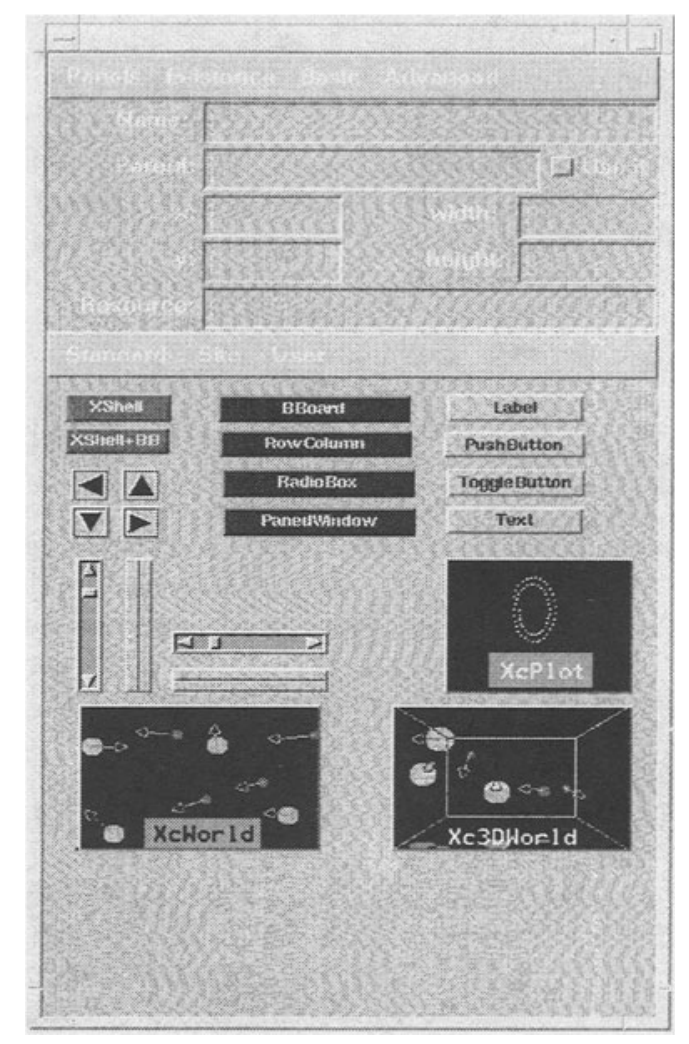

Figure 2. Basic frame of the xdev authoring tool.

An atractive tutorial concerning thermodynamics has been created and supported with xdev-based experiments. The microscopic interpretation of temperature in this course makes easier the understanding of many phenomena involving the pressure in gas and expansion in solids.

Besides this complete tutorial, several other experiments from the domain of physics have been created with xdev.

Considering the current strategy to port all software into Java, a converter (named $x d j$ ) has been developed. It reads the output files created by $x d e v$ and creates corresponding source Java files ( $x \times x$.java).

After that a normal Java compiler creates applets which can be included in hypertext based lessons. The applets use xdj package that are explained later. This conversion process is represented on the following figure.

The basic idea implemented in the converter was to create Java classes (XcWorld, Xc3DWorld) which correspond to the widget components that are implemented in xdev. The functions (callbacks) which were created for the Motif environment are converted into corresponding methods for these Java classes. All these classes are combined in the so called xdj package 
Xdev:

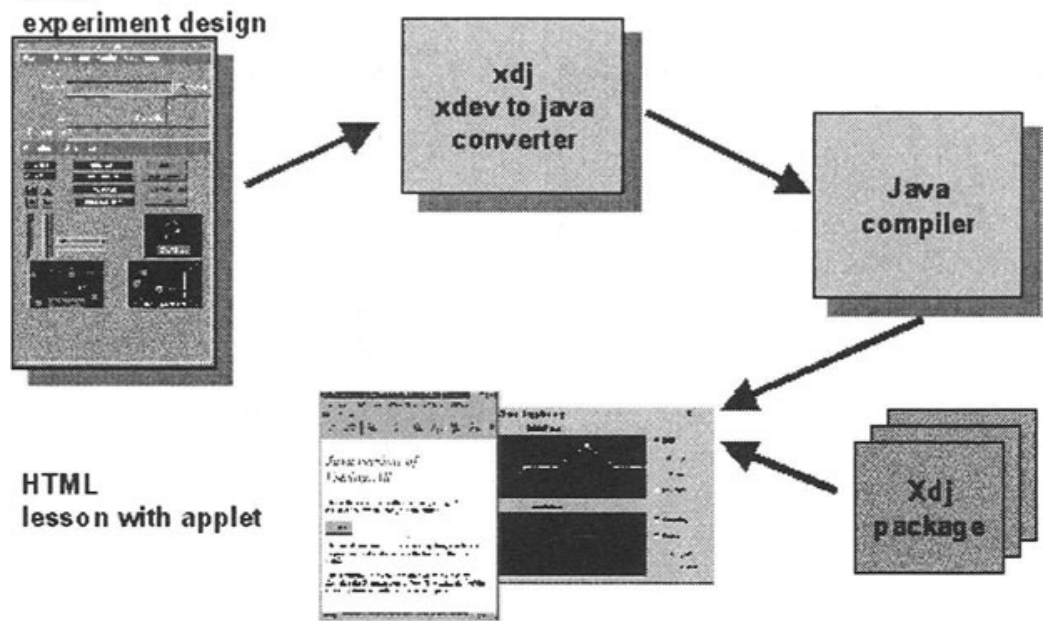

Figure 3. Conversion process of xdev-generated experiments into Java applets.

Figure 4 represents one simple experiment -a vibrating string- that has been converted into a Java applet. The applet is visualized as a pushbutton that is included in the hypertext. On activating this button a new frame with the visualized experiment appears.

The conversion process is $80 \%$ automatic, but some additional manual modifications to the created files are still needed. In any case the programmer's work is simplified and the didactic experience with existing simulation is extended to new platforms.

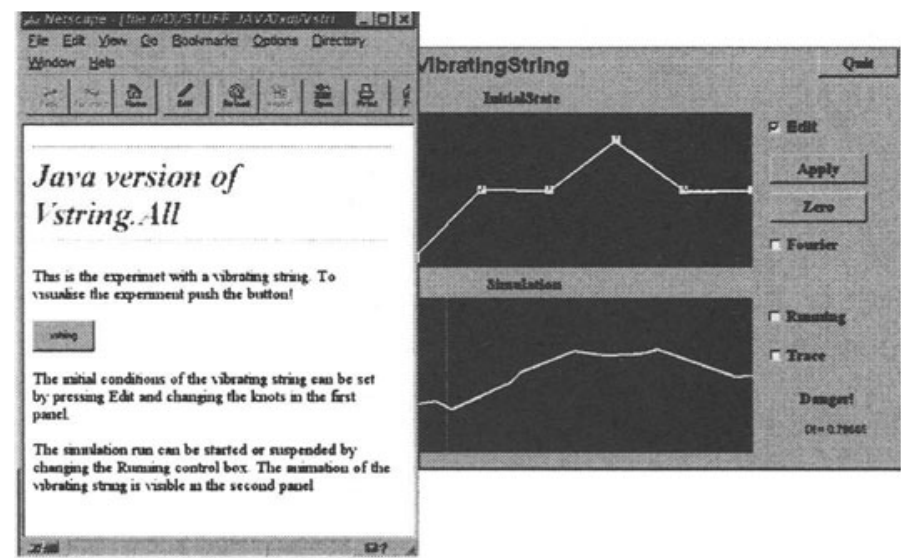

Figure 4. Converted experiment prepared with xdev 


\section{CURRENT DEVELOPMENT}

The user interaction with experiments is achieved through buttons, menus, sliders and checkboxes that are located in the tutorial. Some of these controls have been implemented as separate applets and therefore the concept of simple inter-applet communication has been realized. The applets interact through the variables and methods that are included in a common static abstract class. This approach is simple enough but it is limited to the applets residing on the same HTML page. For the case of separate interacting tutorial pages the communication with sockets was implemented and successfully tested. In the future this interaction will be extended with additional tools which will permit the distributed cooperation of teacher and students. Currently some examples of multiple user interactions through the wellknown whiteboard are being studied. For the moment, the platform in use is still JDK1.02, but the new features offered by JDK1.1 will certainly facilitate this research.

\section{CONCLUSION}

The conversion of physical experiments into java applets offers the required platform independence and provides the possibility of implementing didactical scenarios for virtual classrooms. However, not all simulations can be succesfully converted from the previous Motif compliant environment. First of all, the numerically intensive experiments and visualisations are still too slow. The security restrictions are a factor that inhibits some functions already present in normal applications. The development of robust and stable applications is also limited by the rapid development of new technologies that are therefore sometimes not compatible.

\section{REFERENCES}

Esquembre, F, Zamarro, JM, Martin,E (1992) Authoring Tool Under UNIX/XWindows/MOTIF Oriented to Program Development for Simulation of Physics Phenomena, Computer in Physics, Vol 6, No 4

Haertel, H, Martin, E (1993) New Path for Teaching Electricity, Proc of the Int. Conf on Computer Based Learning, CBLIS 93, Wien

Divjak, S (1996) Conceptual Learning and java programming, Proc of Multimedia and Hypermedia Systems, MIPRO 96, Rijeka

Divjak, S (1996) Conceptual Learning of Sciences Supported by the Simulation of Natural Phenomena, Eurosim, Simulation News Europe, Nov. 1996, ISSN 0929-2268 


\section{BIOGRAPHY}

Sasa Divjak received D.Sc. degree in Electrical Sciences at the Faculty of Electrical and Computer Engineering, University of Ljubljana in 1975. He is head of Chair for Software at the Faculty of Computer and Information Science, Ljubljana, head of Laboratory for Computer Graphics and Multimedia. Responsible for several projects in the field of multimedia and related technologies. Member of Program Council for computer supported education of Slovenian Ministry of Sport and Education. Senior member of IEEE. 